\title{
Re-tessellated Mesh-based Monte Carlo for Wide-Field Illumination Sources
}

\author{
Ruoyang $\mathrm{Yao}^{1}$, Xavier Intes ${ }^{1}$ and Qianqian Fang ${ }^{2}$ \\ ${ }^{1}$ Rensselaer Polytechnic Institute, Department of Biomedical Engineering, $1108^{\text {th }}$ Street, Troy, NY 12180 \\ ${ }^{2}$ Massachusetts General Hospital, Martinos Center for Biomedical Imaging, $14913^{\text {th }}$ Street, Charlestown, MA 02129 \\ fangq@,nmr.mgh.harvard.edu
}

\begin{abstract}
Monte Carlo methods are the gold standard in modeling light propagation through complex turbid tissues. However, with the advent of structured light illumination applications, it is becoming crucial to implement fast and efficient methods to simulate arbitrary wide-field sources over large surface areas. Herein, we improve upon our previous mesh-based Monte Carlo method by enabling the simulation of an extended range of wide-field illumination strategies using a computationally efficient mesh re-tessellation technique.
\end{abstract}

\section{INTRODUCTION}

Optical imaging has become an essential tool in numerous biomedical fields to monitor and quantify the functional, structural and molecular states of thick tissues. Accurately computational models of the light transport inside complex tissue structures are essential for the success of optical imaging techniques, and are playing important roles in the design of novel imaging instrumentation and numerous clinical and preclinical applications. There is a great interest in developing more accurate and computationally efficient forward models. Such interest is recently amplified with the rapid advent of wide-field illumination strategies for spectroscopy [1] or tomography $[2,3]$. Efficient photon transport models that can handle complex illumination strategies are in great needs.

The Monte Carlo method (MC) is recognized as the most accurate approach for simulating photon migration inside general random media, such as biological tissues. By solving the Radiative Transport Equation (RTE) stochastically, MC provides accurate solutions in nearly all media configurations, including the conditions where the diffusion equation fails: such as short source-detector separations, high absorption, low scattering, shallow/small tissues and early-arriving photons [4].With the rapid development of parallel $\mathrm{MC}$ algorithms using modern hardware [5,6,7], MC techniques are no longer restricted to providing reference solutions, but are increasingly used in optimization-based problems $[8,9]$.

Recently, mesh-based formulations have been proposed to further increase $\mathrm{MC}$ computational efficiently and accuracy in the presence of complex boundaries $[10,11]$. However, simulating complex wide-field illumination sources comes at the cost of a computational overhead [12] to the already taxing photon propagation computations. In this work, we built on our previous implementation of wide-field mesh-based forward model [12] to provide an efficient computational tool that allows simulations of complex wide-field sources with a marginal computational overhead.

\section{METHODS}

Our previous approach for handling a wide-field source that is located outside of a mesh domain requires an initial ray-tracing for each launched photon in order to identify the surface injection point [12]. Even for a simplified wide-field source, such calculation could lead to a computation overhead about $5-10 \%$ of the total run-time. In comparison, the ray-tracing calculations inside a tetrahedral mesh are much more efficient because the tetrahedron that encloses the photon is explicitly tracked by the algorithm. Therefore, the key to reduce the overhead due to the initial ray-tracing is to modify the mesh $(M)$ so that an exterior complex source domain $(S)$ becomes part of the modified tetrahedral mesh.

To accomplish this, we propose a mesh re-tessellation algorithm to combine the spatially disconnected source domain, $S$, with the original mesh space, $M$. The source domain, in most applications, is a 3D polyhedron within which the photons are launched. For example, for a planar source with a rectangular profile, $S$ is a simple 3D rectangle characterized by 4 co-planar nodes (shown in Fig. 1a). To merge $S$ and $M$, we first calculate the convex hull for the point-set including all the nodes in $M$ and the vertices in $S$. The convex-hull, $C$, is a triangular surface bounding both $S$ and $M$ (shown in Fig. 1b). Here we assume $S$ is far from $M$ so that $S$ coincides with part of the convex hull $C$. We then tessellate the space, $T$, bounded by the surface $C$ and the exterior surface of $M$. Once the tessellation of $\mathrm{T}$ is completed, we can then merge $T$ with $M$ and form the final modified mesh, M' (see Fig. 1c). The tetrahedral elements in T are specifically labeled and are assumed to have the optical properties of air.

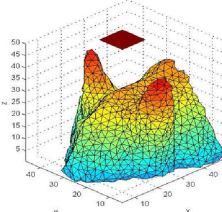

(a)

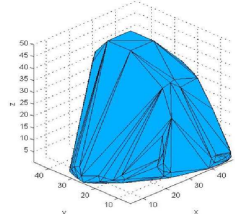

(b)

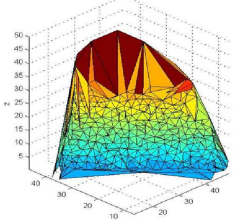

(c)
Fig. 1. (a) Original mesh with a uniform planar source above (b) Convex hull for all nodes in source domain and original mesh (c) Final modified mesh (cross-cut view). 
The final mesh $M^{\prime}$, after the modification, contains a triangulated $S$ at the exterior surface. In the case of Fig. 1, the triangulation of $S$ contains two triangles. When a photon is launched inside $S$, only two point-in-triangle tests are needed to calculate the initial tetrahedron that encloses the photon. Compared to the initial ray-tracing calculations on the original mesh, the computational overhead using the modified mesh is dramatically reduced.

At present our re-tessellated mesh-based Monte Carlo program allows simulation with eleven types of sources, including cone beam, isotropic source, arcsine beam, spatial frequency domain sources and the most important one, 3D quadrilateral arbitrary pattern, which could be powerful when designing and optimizing novel illumination patterns. All source-characterizing information can be represented with no more than 8 floating-points numbers in addition to the source position and incident direction.

\section{VALIDATION}

To evaluate the performance of this improved mesh-based $\mathrm{MC}$ technique, we first tested our new program with a uniform planar source using a widely distributed mouse phantom - the Digimouse model - and compared the results with our previous algorithm. The Digimouse model used in this test contains 42,301 nodes and 210,161 tetrahedron elements (before re-tessellation). For simplicity, uniform optical properties are used: $\mu_{a}=0.3 \mathrm{~cm}^{-1}, \mu_{s}{ }^{\prime}=15 \mathrm{~cm}^{-1}, \mathrm{~g}=0.9, \mathrm{n}=1.37$ derived from the average values for mouse tissues in the NIR spectral region [12]. A uniform square pattern of size $10 \mathrm{~mm}$ by $10 \mathrm{~mm}$ was placed above the model (the range for $x: 15.0-25.0 \mathrm{~mm}, y$ : $50.0-60.0 \mathrm{~mm}, z: 30.0 \mathrm{~mm}$ ) and all photons have an incident direction of [0 0 -1]. After the mesh re-tessellation, 51,053 new elements were created in the extended space. $3 \times 10^{7}$ photons were launched and the fluence rate of 50 time-gates $(100 \mathrm{ps}$ wide) was recorded for each node. The total fluence distribution in logarithmic scale is shown in Fig. 2. There are no noticeable differences between the results of two versions of program.
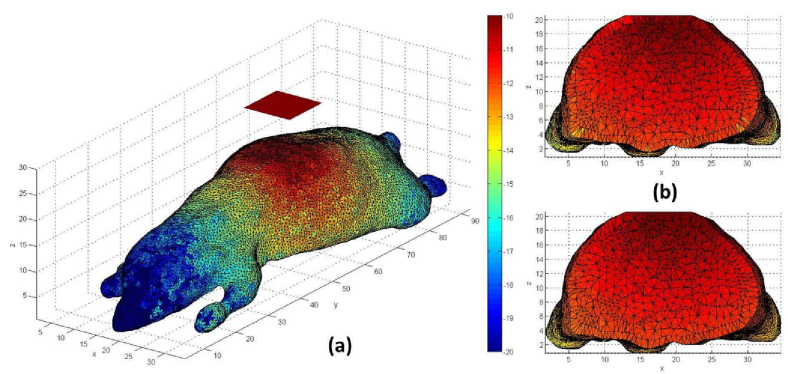

(c)

Fig. 2. Total fluence distribution of digimouse in logarithmic scale under a uniform planar source of $10 \mathrm{~mm}$ by $10 \mathrm{~mm}$

(a) Overall view from the new program

(b) Cross-section view at $y=55 \mathrm{~mm}$ from the new program

(c) Cross-section view at $y=55 \mathrm{~mm}$ from previous version of program

The simulation took about 10 minutes on a personal computer with 12 Intel Core $17-4930 \mathrm{~K}$ processors. Compared to the previous version, where the injection points are calculated by projecting a ray vertically onto the mesh surface, our re-tessellated method achieved a time decrease of about $20 \%$ for computational overhead. Moreover, in the previous version sources are limited to rectangle planar shape and perpendicular to $\mathrm{z}$ axis whereas there are no such limitations in this new program.

\section{CONCLUSION}

We implemented a significantly generalized mesh-based Monte Carlo that supports a wide-range of complex source forms. By dynamically tessellating the mesh to include the source domain, our new approach offers great flexibility and less computational overhead. We reported the initial test results of using a uniform planar source on a Digimouse mesh model. The current implementations lead to a $20 \%$ decrease in the computational overhead associated with wide-field sources modeling. We plan to further extend the current implementation to the detection side for improved performances in wide-field optical tomography based on single-pixel camera strategy [13].

\section{ACKNOWLEDGEMENT}

We greatly appreciate the technical supports of CCI (Center for Computational Innovations) at RPI. This work was partly funded by the National Science Foundation through CAREER award CBET 1149407 and National Institute of Health (NIH) R01 EB19443 and R01 GM114365.

\section{REFERENCES}

[1] O’Sullivan, Thomas D., Albert E. Cerussi, David J. Cuccia, and Bruce J. Tromberg. "Diffuse optical imaging using spatially and temporally modulated light." Journal of biomedical optics 17, no. 7 (2012):

[2] J Chen, V Venugopal, F Lesage and X Intes, "Time Resolved Diffuse Optical Tomography with patterned light illumination and detection," Optics Letters 35, 2121-2123 (2010). PMID: 20596166

[3] V Venugopal, X Intes, “Adaptive wide-field optical tomography,” Journal of biomedical optics 18 (3), 036006-036006 (2013).

[4] Zhu C, Liu Q, "Review of Monte Carlo modeling of light transport in tissues", J Biomed Opt. 2013 May;18(5):50902

[5] J Chen, X Intes, "Comparison of Monte Carlo methods for fluorescence molecular tomography-computational efficiency," Medical physics 38 (10), 5788-5798 (2011)

[6] Fang, Qianqian, and David R. Kaeli. "Accelerating mesh-based Monte Carlo method on modern CPU architectures." Biomedical optics express 3.12 (2012): 3223-3230

[7] Fang, Qianqian, and David A. Boas. "Monte Carlo simulation of photon migration in $3 \mathrm{D}$ turbid media accelerated by graphics processing units." Optics express 17.22 (2009): 20178-20190.

[8] J Chen, X Intes, "Time-gated perturbation Monte Carlo for whole body functional imaging in small animals," Optics express 17 (22), 19566-19579

[9] J Chen, V Venugopal, X Intes, "Monte Carlo based method for fluorescence tomographic imaging with lifetime multiplexing using time gates," Biomedical optics express 2 (4), 871-886

[10] Fang, Qianqian. "Mesh-based Monte Carlo method using fast ray-tracing in Plücker coordinates." Biomedical optics express 1.1 (2010): 165-175

[11] Haiou Shen and Ge Wang, "A study on tetrahedron-based inhomogeneous Monte Carlo optical simulation,” Biomed Opt Express. 2011 Jan 1; 2(1): 44-57.

[12] Chen, Jin, Qianqian Fang, and Xavier Intes. "Mesh-based Monte Carlo method in time-domain widefield fluorescence molecular tomography." Journal of biomedical optics 17.10 (2012): 1060091-1060098

[13] Q Pian, R Yao, L Zhao and X Intes, "Hyperspectral Time-Resolved Wide-Field Fluorescence Molecular Tomography based on Structured Light and Single Pixel-Detection," Optics Letters 40(3), 431-434 (2015) 